\title{
MedienPädagogik
}

Zeitschrift für Theorie und Praxis der Medienbildung

www.medienpaed.com

ISSN 1424-3636

Themenheft Nr. 37: Medienpädagogik als Schlüsseldisziplin in einer mediatisierten Welt. Perspektiven aus Theorie, Empirie und Praxis Herausgegeben von Henrike Friedrichs-Liesenkötter, Lara Gerhardts, Anna-Maria Kamin und Sonja Kröger

\section{Umgangsweisen mit dem Internet empirisch erfassen}

\section{Ausgewählte Methoden und Anwendungsbeispiele für die medienpädagogische Forschung}

Lara Gerhardts

\section{Zusammenfassung}

Angesichts der Omnipräsenz des Internets im heutigen Berufs- und Freizeitalltag eröffnet sich insbesondere auch für die Medienpädagogik ein weites, seit Jahren wachsendes und im ständigen Wandel begriffenes Forschungsfeld. Immer neue Schwerpunktsetzungen auf forschungsthematischer Ebene erfordern zugleich entsprechende Weiterentwicklungen auf Ebene der Forschungsmethodik. Innerhalb der medienpädagogischen Internetforschung ist methodisch derzeit vieles in Bewegung. Um der Entwicklungsdynamik internetbasierter Technologien gerecht zu werden, gilt es, die Methodenentwicklung weiter voranzutreiben. Dafür sollten traditionelle Forschungszugänge und innovative Ansätze gleichermassen in Betracht gezogen und jeweils gegenstandsgerecht zusammengeführt werden. Der Beitrag fokussiert auf empirische Methoden der Datenerhebung, die besonders geeignet erscheinen, um Umgangsweisen mit dem Internet für medienpädagogische Forschungszwecke zu erfassen. Dabei werden sowohl bewährte als auch innerhalb der Disziplin bislang weniger beachtete Verfahren berücksichtigt. Der Methodenüberblick wird um ausgewählte Anwendungsbeispiele ergänzt, die den Mehrwert der einzelnen Verfahren bzw. bestimmter Verfahrenskombinationen veranschaulichen. Der Ausblick zeigt Möglichkeiten und Tendenzen der Weiterentwicklung auf. 


\title{
Collecting Empirical Data on Internet Usage. Selected Methodical Approaches and Examples for Media Pedagogy Research
}

\begin{abstract}
The omnipresence of the Internet in everyday life, whether in professional or leisure activities, opens up a broad and still broadening field of research in the area of media education and pedagogy. This is reflected in the choice of both research topics and methods. Doing Internet research from a pedagogical perspective is a continuously developing field. It is challenging, especially at the methodical level, to keep up with the dynamics of technological change. For future research designs, it is important to consider both traditional and innovative approaches that can be synergized to meet the demands of the respective object of investigation. The paper focuses on empirical methods of data collection on Internet usage that seem particularly beneficial for working on educational and pedagogical research questions in this field. Selected research examples are presented in order to illustrate the added value of different methodical approaches and combinations of methods. Conclusively, possible directions for further development are pointed out.
\end{abstract}

\section{Methodenentwicklung als Desiderat internetbe- zogener medienpädagogischer Forschung}

Infolge der Digitalisierung spielt das Internet inzwischen generationsübergreifend eine wesentliche Rolle für nahezu alle Bereiche der beruflichen und privaten Lebensgestaltung. Der Umgang mit internetbasierten Diensten und Anwendungen ist sowohl Gegenstand als auch Mittel vieler komplexer Lern- und Bildungsprozesse, die sich über verschiedene Nutzer(innen)gruppen hinweg sehr facettenreich gestalten. Für die $\mathrm{Me}-$ dienpädagogik bietet sich dadurch ein weites und hochrelevantes Forschungsfeld, das angesichts rasanter technologischer Fortschritte in ständigem Wandel begriffen ist. 
Um Umgangsweisen mit dem Internet unter medienpädagogisch interessierenden Aspekten erforschen zu können, gilt es, das gängige $\mathrm{Me-}$ thodenrepertoire der Disziplin gleichermassen auf bereichsspezifische Brauchbarkeit wie auch auf «blinde Flecken〉 hin zu reflektieren und die $\mathrm{Me}-$ thodenentwicklung auf dieser Basis zielgerichtet voranzubringen. Methodisch innovative Elemente lassen sich zum einen dadurch gewinnen, dass geeignet erscheinende methodische Ansätze aus anderen Forschungsdisziplinen adaptiert werden. Zum anderen eröffnen die technologischen Weiterentwicklungen auch der Forschung selbst immer grössere Gestaltungsspielräume für methodische Innovation. So können technikseitige Neuerungen entweder dahingehend genutzt werden, dass traditionelle Forschungsmethoden auf zuvor ungekannte Weise medial unterstützt zum Einsatz gelangen; oder auch dahingehend, dass gänzlich neuartige forschungsmethodische Verfahren entwickelt werden, die angesichts neuer Technologien überhaupt erst denk- und umsetzbar werden. Mit dem technologischen Wandel methodisch Schritt zu halten, ist für die Medienpädagogik angesichts ihrer dynamischen, selbst unmittelbar an diesen Wandel geknüpften Forschungsgegenstände besonders entscheidend.

Im Fokus dieses Artikels stehen ausgewählte empirische Methoden der Datenerhebung ${ }^{1}$, die besonders geeignet erscheinen, medienpädagogisch relevante Forschungsdaten zum Internetumgang zu generieren.

Vorangestellt seien zwei begriffliche Klärungen:

- Der Begriff Internetumgang soll hier in einer weit gefassten Bedeutung verwendet werden und sowohl Aspekte der Nutzung (im Sinne handelnden Umgangs) als auch Aspekte des gedanklichen, gefühls- und einstellungsmässigen Umgangs mit dem Internet umfassen.

- Für die Auseinandersetzung mit internetbezogenen Forschungsgegenständen sind grundsätzlich sowohl klassische Verfahren der Präsenzforschung mit ihren jeweiligen Vor- und Nachteilen in Betracht zu ziehen als auch neuere, digital vermittelte Zugangsformen 〈à distance〉,

1 Dieser Beitrag konzentriert sich auf Methoden der Datenerhebung. Für eine vergleichbare Darstellung und Diskussion von Methoden zur Auswertung medienpädagogisch relevanter Forschungsdaten zum Umgang mit dem Internet ist hier nicht der Raum. Eine separate Aufarbeitung wäre im Sinne der weiteren Methodenentwicklung für die medienpädagogische Forschung sicher sinnvoll. 
die häufig als Internetforschung im engeren Sinne (synonym: Onlineforschung) aufgefasst werden. Der Begriff Internetforschung wird in der Fachliteratur jedoch nicht einheitlich verwendet. Internetforschung im weitesten Sinne umfasst jede Art von Forschung mit inhaltlichem oder/ und methodischem Bezug zum Internet (Gnambs und Batinic 2010).

Der Beitrag versteht sich als Überblicksdarstellung, womit aber - angesichts der bereits angesprochenen technikseitigen Entwicklungsdynamik und der damit verbundenen Kreativitätsspielräume für Forschende - keineswegs Anspruch auf Vollständigkeit erhoben wird. Zunächst werden die ausgewählten Erhebungsmethoden systematisiert und in Form von Kurzprofilen einzeln vorgestellt (Punkt 2). Anschliessend wird anhand zweier Anschauungsbeispiele illustriert, wie komplexe Erhebungsdesigns im Bereich der internetbezogenen medienpädagogischen Forschung aussehen können (Punkt 3). Ein kurzer Ausblick auf sich abzeichnende Tendenzen und Möglichkeiten künftiger Methodenentwicklung im Bereich der internetbezogenen medienpädagogischen Forschung rundet den Beitrag ab (Punkt 4).

\section{Internetumgang empirisch erfassen - ein Methodenüberblick für die Erhebung medienpädagogisch relevanter Forschungsdaten}

Die vielfältigen medienpädagogisch relevanten Forschungsgegenstände im Bereich des Umgangs mit dem Internet erfordern ein breitgefächertes Repertoire an empirischen Zugängen. Angelehnt an die erhebungsmethodischen Anregungen, die Carstensen und Winker (2012) für die sozialwissenschaftliche Internetforschung unterbreiten, wird hier vorgeschlagen, 
für internetbezogene Studien mit medienpädagogischem Fokus grundlegend drei Arten von Erhebungsmethoden in Betracht zu ziehen: ${ }^{2}$

(A) Methoden, die den faktischen Umgang mit Internettechnologien auf Ebene des Individuums erfassen, sprich: jene Vorgänge, die bei der einzelnen Internetnutzerin und dem einzelnen Internetnutzer in einschlägigen Anforderungssituationen tatsächlich ablaufen bzw. tatsächlich abgelaufen sind;

(B) Methoden, die entweder subjektive oder aber kollektive Sichtweisen und Wissensbestände zum Internet bzw. zum Umgang mit Internettechnologien erfassen;

(C) Methoden, die medienpädagogisch relevante Rahmen- und Strukturdaten zum Internet bzw. zum Internetumgang erfassen.

Aufgrund der für diesen Beitrag vorgegebenen Umfangslimitierung bedarf es einer Schwerpunktsetzung. So sollen hier ausschliesslich jene Methoden in den Blick genommen werden, die den faktischen Internetumgang auf Ebene des Individuums erfassen (Methodenbereich (A)). Verfahren zur Erhebung subjektiver und kollektiver internetbezogener Sichtweisen und Wissensbestände (Methodenbereich $\left.(B)^{3}\right)$ bleiben in diesem Beitrag

2 Die von Carstensen und Winker (2012) vorgestellte Mehrebenenanalyse bezieht sich inhaltlich auf Fragen sozialer Ungleichheit im Internet. Während die Auswertungskategorien thematisch entsprechend enggefasst sind, erweisen sich die zur Datenerhebung empfohlenen Methoden als weniger spezifisch und prinzipiell für viele Bereiche der empirischen Internetforschung ergiebig. Sämtliche bei Carstensen und Winker angesprochenen Erhebungsmethoden lassen sich - wenngleich von den Autorinnen nicht explizit so systematisiert - den oben unterschiedenen drei Arten von Erhebungsmethoden ((A), (B), (C)) zuordnen.

3 Der hier nicht zu vertiefende Bereich (B) umfasst ein breites Spektrum an Erhebungsmethoden zur Generierung sichtweisen- und wissensbezogener Äusserungen, darunter: Fragebogen, verschiedene Formen mehr oder minder standardisierter Interviews, Gruppendiskussionen und Fokusgruppengespräche sowie Dokumentenanalysen (mit Fokus auf Sichtweisen-/Wissensrekonstruktion); aber auch weniger gängige Verfahren wie Sortier-/Strukturlegetechniken, Constructive Interaction, Stimulated Recalls und TagebuchVerfahren gehören in diesen Bereich. 
aussen vor; ebenso der Bereich der Rahmen- und Strukturdatenerhebung (Methodenbereich $(\mathrm{C})^{4}$ ).

\subsection{Grundsätzliches zur Triangulation}

In Abhängigkeit von Art und Umfang des aktuellen Forschungsvorhabens ist jeweils zu entscheiden, ob es zielführender ist, mit einer ausgewählten Einzelmethode zu arbeiten, um lediglich eine ganz bestimmte Datensorte zu erheben; oder ob sich ein Mehr-Methoden-Design empfiehlt, bei dem verschiedene Erhebungsverfahren eingesetzt und somit verschiedene Datensorten trianguliert werden. Grundsätzlich sei hervorgehoben, dass multimethodische gegenüber monomethodischer Forschung nicht unbedingt zu präferieren ist. Umfassendere und/oder tiefergehende Erkenntnisse über einen Forschungsgegenstand anzustreben, indem dieser aus verschiedenen theoretischen Blickwinkeln betrachtet und methodisch dementsprechend vielschichtig angegangen wird, ist gegenüber einer monoperspektivischen Herangehensweise in jedem Fall deutlich aufwändiger (Flick 2011, 19). Für die Konzeption einer sinnvollen Triangulationsstrategie ist letztlich entscheidend, mit Blick auf den einzuplanenden Aufwand und das erforderliche Know-how von zutreffenden Voraussetzungen auszugehen (Kelle 2001, 206). Der Einbezug jeder weiteren theoretischen Perspektive bzw. jedes dafür erforderlichen methodischen Elements sollte im Sinne der Verhältnismässigkeit von Aufwand und Ertrag genau abgewogen werden. Der folgende Überblick stellt ausgewählte Verfahren für die Gewinnung medienpädagogisch relevanter Daten zum faktischen Internetumgang der einzelnen Nutzerin und des einzelnen Nutzers vor, wobei jeweils - in der hier gebotenen Kürze - auf Potenziale und Limitationen sowie auf praktische Anwendungsbedingungen eingegangen wird. Die Methodenprofile sollen eine grobe Orientierung ermöglichen und somit Auswahl- und Triangulationsentscheidungen im Rahmen des Erhebungsdesigns erleichtern.

4 In den hier nicht zu vertiefenden Bereich (C) fallen Methoden zur Erfassung internetrechtlicher Rahmendaten (Dokumentenanalysen), infrastruktureller Daten zum Internetumgang (standardisierte Befragungen), angebotsstruktureller Daten zum Internet (Webdokumentenanalysen) sowie nutzerstruktureller Daten (Webanalytics). 


\subsection{Methoden zur Erfassung des faktischen Internetumgangs auf Individualebene}

Wann immer es darum geht, Umgangsphänomene zu erforschen, ist generell zu empfehlen, die tatsächliche Praxis des Umgangs einzelner Personen in den Blick zu nehmen. Methoden zur Erhebung der tatsächlichen Umgangspraxis dienen dazu, auch solche Aspekte des Phänomens zu erfassen, die in forschungsgenerierten Äusserungen über eben diese Praxis womöglich zu kurz kommen, gänzlich fehlen oder aufgrund von Darstellungstendenzen stark verzerrt sind. Betroffen sind insbesondere hochgradig automatisierte Vorgänge, die der handelnden Person selbst gar nicht oder zumindest nicht in vollem Umfang bewusst sind; ebenso wie Vorgänge, für die ein hinreichendes Beschreibungsvokabular fehlt; oder auch Umgangsaspekte, über die nicht gern offen gesprochen wird, etwa weil sie als normabweichend, ungeschickt o.ä. angesehen werden. Speziell was den Umgang mit technisch-medialen Gegenständen anbelangt, weisen Carstensen und Winker (2012) darauf hin, dass sich durch die Beobachtung der tatsächlichen Aktivitäten einzelner Nutzerinnen und Nutzer vieles erkennen lasse, was in deren Selbstberichten offen oder kryptisch bleibe. Unter Verweis auf eigene frühere Studien resümieren die Autorinnen:

«Es hat sich gezeigt, dass insbesondere im Umgang mit neuen Technologien oftmals die konkrete Sprache fehlt, also Begrifflichkeiten und Formulierungen fehlen, um alltägliche Routinen und Tätigkeiten sowie ungeübte Handlungen zu beschreiben.» (ebd., 14)

Wie einzelne Personen mit einer bestimmten Anforderung faktisch umgehen, lässt sich grundsätzlich auf zwei Wegen untersuchen: zum einen anhand situativ erhobener Prozessdaten, die die jeweils interessierenden individuellen Aktivitäten innerhalb eines definierten Zeitraums fortlaufend abbilden; und zum anderen anhand postsituativ erhobener Produktdaten, in denen sich zuvor stattgefundene Prozesse individueller Anforderungsbewältigung dokumentieren. Auch der faktische Umgang mit Internettechnologien kann in diesem Sinne sowohl prozess- als auch produktbasiert erforscht werden. Im Folgenden wird zunächst auf die Erfassung von Prozessdaten des Internetumgangs einzelner Nutzerinnen und Nutzer 
eingegangen; und anschliessend auf die Erfassung von Produktdaten zur Rekonstruktion bestimmter Nutzer(innen)aktivitäten.

\subsubsection{Erfassung von Prozessen des Internetumgangs einzelner Nutzerinnen und Nutzer}

Prozessdaten werden erhoben, während die für das jeweilige Umgangsphänomen charakteristischen Vorgänge gerade ablaufen. Bezogen auf den Umgang mit Internettechnologien bedeutet dies, Situationen zu beobachten und aufzuzeichnen, in denen eine Probandin oder ein Proband gerade in der jeweils interessierenden Weise im Internet aktiv ist (also z.B. eine Internetrecherche durchführt, einen Beitrag auf einer Social Media Site postet, den eigenen Blog pflegt, sich mit einem Online-Lernangebot beschäftigt, ...). Prozessdaten bieten den Vorteil, tatsächliche Vorgänge über eine gewisse Zeitspanne kontinuierlich abzubilden, und erlauben somit eine direkte (soll heissen: zeitgleiche) Beobachtung. Was die Erhebung von Prozessdaten betrifft, ist grundlegend zu differenzieren zwischen Verfahren zur Erfassung äusserlicher Vorgänge einerseits und Verfahren zur Erfassung innerlicher Vorgänge andererseits.

(a) Erfassung äusserlicher Prozesse des Internetumgangs einzelner Nutzerinnen und Nutzer

Zu den äusserlichen Prozessen zählen in diesem Zusammenhang all jene, die sich an der Körperoberfläche und im unmittelbaren Austausch mit der umgebenden Aussenwelt abspielen. Für die empirische Sozialforschung können je nach Forschungsfrage grundsätzlich alle Bewegungsabläufe sowie gestischen, mimischen und verbalen Ausdrucksweisen interessant sein, die der Interaktion mit einer anderen Person, einem technischen oder auch nicht-technischen Gegenstand dienen. Zu den äusserlichen Prozessen im Umgang mit Internettechnologien zählen im engeren Sinne zunächst einmal die Bedienung von Tastatur und Maus bzw. die Tipp- und Wischbewegungen an Touch Screens; die Fortbewegung auf einer Webseite und zwischen verschiedenen Webseiten; und auch die Art und Weise der Betrachtung von Bildschirminhalten sowie Mimik und Gestik der auf den Bildschirm schauenden Person(en) fallen in diesen Bereich. Hinzu kommen 
verschiedene Arten von verbalen Äusserungen, darunter z.B. schriftliche oder mündliche Eingaben beim Stellen einer Suchanfrage bzw. bei Direktaufruf bestimmter Webseiten; vor allem aber auch jegliche Verbalisierungen, die der synchronen oder asynchronen Online-Kommunikation mit anderen Personen dienen. Sofern die Forschungsfrage es nahelegt und der Beobachtungsausschnitt entsprechend gross gewählt wird, zählen zu den äusserlichen Prozessen des Internetumgangs in einem erweiterten Sinne auch noch verschiedene Vorgänge, die parallel zum eigentlichen OnlineGeschehen (also offline) verlaufen, jedoch eng mit diesem verknüpft sind und sich entsprechend auswirken. Beispiele für begleitende Offline-Aktivitäten mit mehr oder minder starkem Einfluss sind paralleles Telefonieren oder gar Fernsehschauen; oder auch etwaige auf die Internetnutzung bezogene Unterstützungshandlungen seitens Dritter (in schulischen oder beruflichen Kontexten z.B. Hilfestellungen von Mitschülerinnen und Mitschülern bzw. von Kolleginnen und Kollegen; und im häuslichen Umfeld vor allem der informelle Austausch zwischen Familienmitgliedern).

Generell gilt, dass all diese äusserlichen Prozesse des Internetumgangs einer unmittelbaren Fremdbeobachtung durch den Forschenden zugänglich sind (d.h. die Beobachtbarkeit ist anders als bei innerlichen Aspekten sofort gegeben, ohne dass zuvor irgendeine Art von «Übersetzung〉 in ein beobachtbares Format stattgefunden haben muss, vgl. in Abgrenzung Punkt (b)). Je nach Forschungsfrage kann entweder bereits eine einfache Beobachtung mit blossem Auge und eine Protokollierung bzw. Aufzeichnung mittels simpler Technik aufschlussreiche Forschungsdaten liefern; oder es bedarf der Nutzung komplexerer Beobachtungs- und Aufzeichnungsgeräte, die eine starke optische Vergrösserung, eine akustische Verstärkung, spezielle Zeitlupen-Aufnahmen usw. ermöglichen. Mit Hilfe derartiger Funktionen lassen sich dann auch Teilprozesse beobachten und aufzeichnen, die angesichts ihrer Feindifferenziertheit, ihrer geringen Hörbarkeit, ihrer hohen Ablaufgeschwindigkeit o.ä. unterhalb der Schwelle menschlicher Sinneswahrnehmung ablaufen. Für jegliche Form der Prozessbeobachtung bzw. -aufzeichnung gilt es, deren spezifische, allein schon technikbedingte Perspektivität und Selektivität zu reflektieren. So ist stets mitzudenken, dass auch die vermeintlich 〈objektiven〉 Beobachtungsdaten 
zu äusserlich ablaufenden Prozessen ein Stück weit konstruiert sind (Döring und Bortz 2016, 328).

Um medienpädagogisch relevante Prozessdaten zu äusserlichen Aspekten des Umgangs mit Internettechnologien zu erheben, scheinen vier Verfahren besonders geeignet:

Ethnographische Feldbeobachtung

Die ethnographische Feldbeobachtung sowie begleitend und retrospektiv angefertigte Feldnotizen liefern besonders komplexe Daten zu äusserlich ablaufenden Prozessen im Umgang mit Internettechnologien und ermöglichen darüber hinaus, deren Eingebundenheit in übergeordnete lebensweltliche Zusammenhänge zu erschliessen. Anstatt eine Fokussierung auf einzelne, vorab festgelegte Aspekte internetbezogenen Handelns vorzunehmen, wird die Beobachtungseinheit hier sehr breit definiert: Es geht darum, die interessierenden Interaktionsabläufe als Gesamtphänomen inklusive der bedingenden Kontextfaktoren in den Blick zu nehmen. Zu diesem Zweck taucht die Forscherin oder der Forscher als teilnehmende Beobachterin bzw. teilnehmender Beobachter in die soziale Wirklichkeit der Beforschten ein und erlebt das Feldgeschehen aus nächster Nähe mit. Dabei wird eine gering strukturierte Form wissenschaftlicher Beobachtung und Protokollierung realisiert, um ein hohes Mass an Offenheit zu wahren und insbesondere auch unerwartete Vorkommnisse im Feld zu erfassen. Exemplarisch sei hier auf die Dissertation von Seib (2006) verwiesen, die umfangreiche teilnehmende Beobachtungen in der Grundschule durchgeführt hat, um die Internetnutzung von Kindern unter möglichst natürlichen schulischen Bedingungen, d.h. eingebettet in die typischen Routinen und Interaktionen im Rahmen des Unterrichtsgeschehens, erfassen zu können. Seit geraumer Zeit bildet auch das Internet selbst einen eigenen «Kulturraum» und somit ein für ethnographische Forschungen unerschöpfliches Feld (Marotzki 2003). Online-ethnographische Feldbeobachtungen zielen meistens auf die Untersuchung virtueller Communities (ebd.). Ein interessantes Beispiel aus diesem Bereich liefern Ducheneaut und Moore (2005) mit ihrer Studie zur Entwicklung sozialer Kompetenzen unter Spielern von Multiplayer Online Games. 
Wird eine ethnographische (Offline- oder Online-)Beobachtung zur Datenerhebung in Betracht gezogen, ist zu bedenken, dass es sich um eine sehr voraussetzungsvolle Methode handelt. Die grundlegende Anwendungsbedingung ist, dass die Forscherin oder der Forscher mit der Ethnographie als qualitativer Forschungsstrategie in ihrer Gesamtheit vertraut ist, um den beobachtenden Zugang sinnvoll mit weiteren ethnographischen Erhebungsmethoden kombinieren zu können und komplexen Forschungsgegenständen gerecht zu werden. ${ }^{5}$ Des Weiteren ist zu bedenken, dass als ethnographische Beobachterinnen und Beobachter nur felderfahrene Personen mit einer grossen Aufmerksamkeitsspanne in Frage kommen, die den besonders umfänglichen und wenig vorstrukturierten Beobachtungsanforderungen gewachsen sind. Zudem bedarf es bei dieser Form des Beobachtens einer ausgeprägten Empathie- und Anpassungsfähigkeit, um verwertbare Forschungsdaten zu generieren. Entscheidend ist, sich als teilnehmende Beobachterin oder teilnehmender Beobachter möglichst unauffällig ins Feldgeschehen einzufügen und die natürlichen Abläufe nicht zu stören. Dazu gehört auch, stets kontextsensibel zu entscheiden, ob der Einsatz ergänzender Foto-, Audio-, und Videoaufnahmen die Beobachtungsdaten bereichert oder aber deren ökologische Validität durch eine technische Überformung der Situation gefährdet wird (Kelle 2001). Gelingt es, als Feldforscherin oder Feldforscher situativ das rechte Mass an Beteiligung zu finden, weichen anfangs eventuell auftretende Irritationen der regulären Feldakteurinnen und -akteure sehr schnell der Gewohnheit, sodass sich beobachterbedingte reaktive Effekte in Grenzen halten. Generell ist ein vergleichsweise hoher Zeit-/Kostenaufwand für Datenerhebungen mittels ethnographischer Beobachtung einzukalkulieren: Je nach Vorerfahrung bedarf es ggf. zunächst intensiver Beobachter(innen) schulungen. Sodann folgt eine vergleichsweise langwierige Beobachtungsphase im Feld, weil kürzere Beobachtungen eben die spezifisch ethnographische Mitbetrachtung lebensweltlicher Kontexte kaum zulassen. Auch ist im Rahmen eines ethnographischen Zugangs bereits während der

5 Neben ethnographischen Beobachtungen stellen sog. Feldgespräche als spezielle Form des qualitativen Interviews (Methodenbereich (B) - hier nicht zu vertiefen, vgl. Punkt 1) sowie die Sammlung von Dokumenten und Artefakten (vgl. Punkt 2.2.2 in diesem Teilkapitel) weitere Möglichkeiten der Datengewinnung im Bereich der ethnographischen Feldforschung dar. 
Datenerhebung besonders viel Zeit für kollegiale Inter- oder Supervision einzuplanen, um vorzeitigen Verengungen bei der Feldbeobachtung systematisch vorzubeugen.

Weniger geeignet ist ein ethnographischer Zugang immer dann, wenn tiefergehende Informationen über einen ganz bestimmten Teilaspekt des Umgangs mit dem Internet generiert werden sollen und die Peripherie dieses Teilaspekts nicht weiter interessiert. Sehr offene Beobachtungen gehen stets mit der Möglichkeit einher, dass zu einzelnen Aspekten wider Erwarten gar nicht viel zu beobachten ist, weil das beobachtete Geschehen einen unerwarteten Verlauf nimmt. Sind also spezifische Informationen zu einem ganz bestimmten, bereits klar definierten Aspekt des Internetumgangs gefragt, empfiehlt es sich, den Fokus von vornherein sehr viel enger auszurichten als bei ethnographischen Beobachtungen gewollt und Lebensweltbezüge gezielt aus der Datenerhebung auszuklammern.

Im Folgenden werden drei Verfahren vorgestellt, die unter der Bedingung eines vergleichsweise engeren Blickwinkels geeignet sind, bestimmte äusserliche Abläufe im Umgang mit dem Internet zu erfassen:

\section{Aufzeichnung von Logfiles}

Bei Logfiles handelt es sich um digitale, während einer Mensch-Computer-Interaktion automatisiert erzeugte Protokolldateien, die sowohl systemseitige Ereignisse als auch Aktivitäten der Nutzerinnen und Nutzer erfassen können. Ihrem ursprünglichen Zweck gemäss dienen Log-Daten der Fehlerermittlung und -behebung bei Systemabstürzen, bei Konnektivitätsproblemen in digitalen Netzwerken usw. Seit geraumer Zeit werden sie aber auch für die Erforschung von Nutzer(innen)verhalten in digitalen und insbesondere in Internetumgebungen herangezogen. Letzteres ist für grundlagen- wie auch anwendungsorientierte Studien in verschiedenen Disziplinen interessant, insbesondere für die Online-Marktforschung, die Usability-Forschung und nicht zuletzt auch für Studien im Bereich der Medienpsychologie und Medienpädagogik. Logfiles können unterschiedlich viele Arten von system- und nutzer(innen)seitigen Vorkommnissen erfassen, je nachdem welche standardmässigen Voreinstellungen vorhanden sind bzw. welche Aspekte im Falle einer gezielten Programmierung als relevant definiert wurden. Was die nutzer(innen)seitigen Aktivitäten betrifft, 
enthalten Logfiles in aller Regel mindestens Einträge zu jedem Seitenaufruf bzw. -wechsel samt der zugehörigen Zeitwerte. Darüber hinaus besteht bei Logfile-Aufzeichnungen an lokalen Rechnern heutzutage die Möglichkeit, noch sehr viele weitere Aktivitäten kontinuierlich zu erfassen, etwa die Mausbewegungen, Klicks und sämtliche Tastatureingaben. Bezogen auf den Umgang mit dem Internet ermöglichen Logfiles die Untersuchung von Nutzer(innen)verhalten aus zwei unterschiedlichen Perspektiven, die beide auch für medienpädagogische Kontexte bedeutsam sind: Zum einen kann eine angebotszentrierte, zum anderen eine rezeptionszentrierte Untersuchungsperspektive eingenommen werden. In Abhängigkeit davon erweisen sich unterschiedliche Arten von Logfiles als zweckdienlich. Während für angebotszentrierte Studien vor allem serverbasierte Logfiles in Frage kommen, sind in der rezeptionszentrierten Forschung clientseitig registrierte Logfiles angezeigt (vgl. zur Vertiefung: Welker 2019).

Bei angebotszentrierten Untersuchungen geht es darum, Nutzungsprozesse innerhalb eines bestimmten Webangebots zu erfassen, um so zu eruieren, ob die inhaltliche, sprachliche und ästhetische Gestaltung bei der adressierten Nutzer(inneng)ruppe die intendierte Wirkung erzielt. Als zentrales Instrument im Bereich der Webanalytics spielen Logfiles in solchen Untersuchungen eine wichtige Rolle. Die erhobenen Log-Daten werden softwaregestützt aggregiert, sodass hier letztlich nicht mehr mit den Prozessdaten selbst, sondern mit daraus berechneten überindividuellen Kennwerten weitergearbeitet wird.

Rezeptionszentrierte Untersuchungen zielen hingegen darauf, Nutzungsprozesse einer bestimmten bzw. mehrerer bestimmter Person(en) über mehrere Webangebote hinweg zu erfassen und somit exakt nachzuvollziehen, wie die betreffende(n) Person(en) vorgehen und welche Interessen, Vorlieben und Kompetenzen bzw. auch Schwierigkeiten dabei deutlich werden. Hier wird fallbezogen mit den Prozessdaten gezielt gesampelter Einzelfälle gearbeitet, um aus diesen Daten softwaregestützt bestimmte Kennwerte zur Charakterisierung des individuellen Nutzungsverlaufs zu ermitteln. Grundlegend lassen sich atomistische Kennwerte (zu Häufigkeit und Dauer einzelner Seitenaufrufe) und sequenzielle Kennwerte (zu Häufigkeit und Gestalt bestimmter Folgen von Seitenaufrufen) unterscheiden. Während erstere zunächst einmal gewisse Rückschlüsse auf 
die allgemeine Aufmerksamkeitsallokation erlauben, können letztere als mögliche Anhaltspunkte für Kohärenz und Gründlichkeit der Informationsverarbeitung in hypermedialen Umgebungen gelten (z.B. indizieren hohe Kennwerte im Bereich linearer Sequenzen ein systematisches $\mathrm{Na}$ vigationsverhalten; hohe Kennwerte im Bereich non-linearer Sequenzen, sog. 〈Backtracks〉, können hingegen auf unsystematisches Surfen, Orientierungsschwierigkeiten etc. hindeuten) (vgl. für Details zu den Kennwerten individuellen Nutzer(innen)verhaltens und ihrer Interpretation: Richter u. a. 2005). Auf Basis solcher Kennwerte lassen sich dann z.B. die Erfolgsbilanzen verschiedener Navigationsverläufe (gemessen an erfolgreich lokalisierten Informationen, an erfolgreich gelösten Aufgaben o.ä.) miteinander vergleichen, zu weiteren Untersuchungsaspekten in Beziehung setzen und dergleichen mehr.

Logfiles zur Erforschung von Nutzer(innen)verhalten in hypermedialen Umgebungen einzusetzen, hat mehrere Vorzüge. Da Log-Daten im Zuge von Mensch-Computer-Interaktionen auch unter natürlichen Bedingungen generiert werden und dies unbemerkt im Hintergrund geschieht, geht die Nutzung zu Forschungszwecken allenfalls mit einem geringen Reaktivitätsrisiko einher. Selbst wenn im Vorhinein spezielle Programmierungen vorgenommen werden, um die Aussagekraft der Logfiles für den jeweiligen Forschungszweck zu erhöhen, wird damit nicht in die natürliche Interaktion eingegriffen. Insbesondere bei personenbezogenen Untersuchungen an lokalen Rechnern sind die Forschungsteilnehmerinnen und -teilnehmer aus forschungsethischen Gründen selbstverständlich bezüglich der Logfile-Registrierung zu informieren und um Einverständnis zu bitten. Die Erfahrung zeigt jedoch, dass dies mehrheitlich nicht zu anhaltenden Verhaltensverzerrungen führt, sondern Probandinnen und Probanden sich sehr schnell in ihre Aufgabe vertiefen und ein natürliches Vorgehen an den Tag legen. Des Weiteren liefern Logfiles dem Forschenden ungeachtet der Dauer des Beobachtungszeitraums exakte, lückenlose und objektive Zeitreihen- und Aktivitätsdaten, die ausserdem sofort übersichtlich strukturiert ausgegeben werden. Die maschinell erzeugten Protokolle sind klassischen Beobachtungsprotokollen in puncto Genauigkeit und Arbeitsökonomie klar überlegen. Exemplarisch sei auf eine medienpädagogisch hochrelevante Untersuchung von Feil, Gieger, und Grobbin (2013) 
hingewiesen, die u.a. anhand umfangreicher Log-Datensätze die Suchmaschinennutzung von Kindern untersucht haben.

Der zeitliche und finanzielle Aufwand für die Datenerhebung als solche ist aufgrund der automatisierten Generierung und Speicherung der Log-Dateien gering. Die Forscherin oder der Forscher sollte aber Zeit einplanen, um sich zuvor mit den technischen Grundlagen so weit vertraut zu machen, dass die für den jeweiligen Forschungskontext ergiebigste technische Lösung gewählt und ggf. eine bedarfsgerechte Programmierung vorgenommen werden kann.

Logfile-Registrierungen für Forschungszwecke stossen dort an ihre Grenzen, wo es darum geht, nicht nur die Navigationsbewegungen zu erfassen, sondern tiefere Einblicke in kognitive und/oder emotionale Verarbeitungsprozesse zu gewinnen. So lässt sich aus Log-Daten nicht entnehmen, welche Inhalte jeweils aktuell im Bildschirmausschnitt zu sehen sind; geschweige denn, welche davon die Probandin bzw. der Proband wie genau verarbeitet, an welchen Stellen die Verarbeitung zwischenzeitlich unterbrochen wird und welche Teilbereiche gänzlich ausser Betracht bleiben. Unklar bleibt auch die Ergebnisqualität der Informationsverarbeitung, d.h. ob ein hinreichendes Verständnis erzielt werden konnte oder womöglich auf Basis un-/missverstandener Informationen weitergearbeitet wird. Des Weiteren fehlen Kontextinformationen, um sich einen Gesamteindruck von der untersuchten Person zu verschaffen und einzelne Log-Ereignisse vor diesem Hintergrund zuverlässiger interpretieren zu können. So lässt sich beispielsweise nichts über das Befinden während der medialen Interaktion oder über etwaige situative Störfaktoren aussagen. Ebenso wenig erfährt man über generelle Sichtweisen und Wissensbestände der Person, die ggf. die ein oder andere Navigationsentscheidung erhellen könnten. Identifizierte Bewegungsmuster wie etwa die oben angesprochenen «Backtracks〉 bleiben für sich genommen somit recht unspezifisch: Zwar mögen sie überwiegend auf unsystematisches Navigationsverhalten hindeuten. Dennoch sind durchaus Kontexte vorstellbar, in denen sie vielmehr als sinnvolles Explorations- oder auch Absicherungsverhalten anzusehen sind (Richter u. a. 2005). Schliesslich fehlen in Log-Daten auch sämtliche Navigationsereignisse, die von dem jeweiligen Logfile-Typ nicht standardmässig erfasst bzw. gezielt zur Registrierung einprogrammiert wurden. Aspekte 
des Bildschirmgeschehens, die sich womöglich erst im Verlauf einer Untersuchung als relevant herauskristallisieren und daher zuvor nicht bedacht wurden, wie z.B. versehentliches Daneben-Klicken, suchende Bewegungen mit der Maus o.ä., lassen sich dann später nicht mehr nachvollziehen. Da Logfiles viele deutungsrelevante Aspekte des Gesamtgeschehens nicht erfassen, ist generell eine gezielte Kombination mit weiteren Erhebungsverfahren zu empfehlen.

(Kombinierte) Bildschirm- (und Personen-)Videographie

Prozesse des Internetumgangs einzelner Nutzerinnen und Nutzer lassen sich auch videographisch erfassen. Im Mittelpunkt dieser 〈Spezialform〉 der Videographie steht das auf dem Bildschirm sichtbare Interaktionsgeschehen zwischen Nutzerin bzw. Nutzer und System. Mittels Bildschirmaufzeichnung lassen sich sämtliche in der Bildschirmansicht zu erkennende Nutzer(innen)aktivitäten (d.h. Mausbewegungen, Bewegungen der Scroll-Leisten, Klicks, Tastatureingaben) sowie die dadurch initiierten Änderungen der Bildschirmansicht (z.B. das Erscheinen von DropdownMenüs, temporäre Textmarkierungen und vor allem Seitenwechsel) über den gesamten Verlauf einer Internetsitzung aufzeichnen und beobachten. Meist ist es sinnvoll, synchron zur Bildschirmvideographie in einem separaten Personenvideo auch noch die Internetnutzerin bzw. den Internetnutzer selbst aufzunehmen. Die Personenvideographie liefert in diesem Zusammenhang ergänzende Daten zu Mimikveränderungen während der Internetsitzung und, je nach Einstellungsgrösse, ggf. auch zu Gestik, Körperhaltung und zum situativen Kontext der Aufnahme. Da auch eine Audiospur aufgezeichnet werden kann und sollte, lassen sich zudem Begleitkommentare der untersuchten Person aufnehmen (die natürlicherweise meist nur vereinzelt vorkommen, aber z.B. im Rahmen der Methode des Lauten Denkens auch systematisch miterhoben werden können (vgl. Punkt (b)).

Die Kombination aus Bildschirm- und Personenvideographie ist technisch auf unterschiedlichen Wegen umsetzbar. In sozialwissenschaftlichen Forschungskontexten wurde bis vor wenigen Jahren häufig noch mit einfacher Videotechnik gearbeitet (z.B. Irion 2008): Dabei filmte die erste Kamera, hinter der Untersuchungsperson positioniert, den Bildschirm ab; 
die zweite Kamera filmte die Person von vorn. Bei dieser Vorgehensweise erwies sich aber die mangelnde Bildqualität als problematisch; auch gingen die auffälligen Kameraaufbauten mit einem erheblichen Reaktivitätsrisiko einher. Nachteilig war schliesslich auch der erhebliche Aufwand bei der späteren Zusammenführung und Synchronisation der beiden getrennt generierten Videospuren. Inzwischen greift man bevorzugt auf SoftwareLösungen, sogenannte Screencasting Software, zurück. Die Software liest die Bildinformationen kontinuierlich direkt aus dem Arbeitsspeicher und erzeugt aus diesen Daten eine Videodatei. Eine entsprechend komplexe Software kann parallel über die Webcam bzw. das interne Mikrofon des Computers auch das Personenvideo samt Audiospur aufnehmen. Durch die Aufnahme mittels Software entfällt der Arbeitsschritt des nachträglichen Synchronisierens der so erzeugten Video- und Audiodateien.

Generell bietet Videographie den grossen Vorteil, gerade auch langdauernde Prozesse 〈naturgetreu〉 - d.h. vergleichsweise objektiv und vollständig - festzuhalten und somit das komplexe Gesamtgeschehen seiner Flüchtigkeit zu entheben. Im Gegensatz zu Beobachtungsprotokollen, die von einer menschlichen Beobachterin bzw. Beobachter in Echtzeit erstellt werden, sind Videodaten um ein Vielfaches weniger selektiv und kommen somit den tatsächlichen Abläufen deutlich näher. Die Tatsache, dass Videoaufzeichnungen kaum durch forscher(innen)seitige Vorselektionen beeinträchtigt sind und auch im weiteren Forschungsverlauf jederzeit erneut auf das hochkomplexe Videomaterial zurückgegriffen werden kann (um erste Interpretationen und Hypothesen abzusichern, nach weiterführenden Hinweisen zu suchen usw.), prädestiniert videographische Erhebungsmethoden in besonderem Masse für qualitative, auf Offenheit und Zirkularität basierende Forschungsdesigns (Irion 2008, 127). Die Sonderform der Bildschirmvideographie ist darüber hinaus mit weiteren spezifischen Vorteilen verbunden, was die Erfassung von Mensch-Computer-Interaktion betrifft: Erstens lassen sich durch Videoaufnahmen des Bildschirms die Inhalte und das Design der jeweils aufgerufenen Seiten festhalten (und zwar exakt so, wie sich beides zum Zeitpunkt der zu analysierenden Internetsitzung dargeboten hat, womöglich schon kurze Zeit später online gar nicht mehr verfügbar ist). Videodaten gewährleisten, dass dauerhaft nachvollziehbar bleibt, mit welchen Web-Inhalten und welchen Web-Designs die 
jeweilige Nutzerin bzw. der jeweilige Nutzer tatsächlich konfrontiert war und welche Selektionsentscheidungen sie oder er folglich treffen musste. Zweitens werden nicht nur bestimmte, sondern alle sichtbaren Vorgänge zwischen Nutzerin bzw. Nutzer und Interface erfasst: So sind anhand der Videoaufzeichnung auch solche Vorgänge beobachtbar, die etwa in Logfiles gar nicht als Ereignis ausgewiesen werden, weil sie letztlich nicht zu einer Reaktion des Systems geführt haben. Je nach Forschungsanliegen können gerade auch solche systemseitig nicht identifizierbaren Aktionen relevant sein (darunter z.B. versehentliche Fehlklicks neben den eigentlichen Hotspot; das Klicken auf dekorative Elemente, die fälschlich für Bedienelemente gehalten werden usw.). Die Kombination mit Personenvideographie und Audioaufzeichnung bietet einen weiteren entscheidenden Vorteil: Durch die Beobachtung der Körpersprache der Untersuchungsperson, deren verbale Äusserungen und auch durch etwaiges, von der Personenkamera mit eingefangenes Kontextgeschehen (wie z.B. die parallele Nutzung analoger Medien, unterstützende Aktivitäten anderer Personen, auftretender Störfaktoren usw.) lässt sich ein Gesamteindruck von der Erhebungssituation gewinnen, der es oftmals erleichtert, die aus der Bildschirmvideographie verfügbaren Verhaltensdaten zutreffend einzuordnen. Wo beispielsweise anhand des Bildschirmvideos einer Internetrecherche lediglich ein mehrfaches Hin- und Herspringen zwischen verschiedenen Webseiten feststellbar ist, lässt sich mit Hilfe des Personenvideos unter Umständen klären, ob diese 〈Backtracks〉 (s.o.) im jeweiligen Fall als Ausdruck von Orientierungslosigkeit zu deuten sind (weil videographierte Person hektisch wirkt, die Hände über dem Kopf zusammenschlägt o.ä.); oder ob die schnellen Seitenwechsel vielmehr im Rahmen eines gewissenhaften Gesamtvorgehens auftreten und dem gezielten Vergleich von Informationen dienen (z.B. vor dem Hintergrundszenario, dass die videographierte Person parallel mit einer anderen Person über die Glaubwürdigkeit von Online-Informationen diskutiert).

Ein frühes und auch aus medienpädagogischer Sicht interessantes Anwendungsbeispiel einer kombinierten Bildschirm- und Personenvideographie stammt aus der fachdidaktischen Forschung zum Medieneinsatz im Deutschunterricht. Seib (2006) analysiert unter Einsatz der Screencasting Software Lotus ScreenCam den informationsorientierten Internetumgang 
von Grundschulkindern. Ein weiteres Beispiel findet sich bei Gerhardts (in Vorb.): Hier wird mit Hilfe der Software Camtasia eine kombinierter Bildschirm- und Personenvideographie mit Sekundarschülerinnen und -schülern realisiert, um didaktisch relevante Komponenten des Konstrukts Internetrecherchekompetenz zu ermitteln (vgl. auch Punkt 3).

Was den einzuplanenden Aufwand und das erforderliche Know-how betrifft, ist zu bedenken, dass inzwischen eine Vielzahl an Software-Angeboten mit grosser Preisspanne auf dem Markt ist. Es ist lohnenswert, etwas Zeit zu investieren, um Preis-Leistungsverhältnisse zu vergleichen und so letztlich die für den jeweiligen Forschungszusammenhang günstigste Lösung mit allen benötigten Funktionen ausfindig gemacht werden kann. Anschliessend gilt es, sich mit der gewählten Software umfassend vertraut machen und ggf. sogar den technischen Gesamtaufbau genau einzuüben, um später in der Erhebungssituation zeitsparend und flexibel agieren zu können. Der Zeitaufwand für die Vorbereitung des Erhebungssettings ist ebenfalls nicht zu unterschätzen. Insbesondere wenn an verschiedenen Orten (z.B. in verschiedenen Schulen oder in häuslichen Kontexten) erhoben werden soll und die gesamte Technik jedes Mal verlagert und erneut aufgebaut werden muss, kommen in Summe viele Stunden zusammen. Zwar lässt sich der Technikaufbau durch Nutzung einer Screencasting Software im Vergleich zur klassischen Videovariante erheblich beschleunigen. Dennoch kann es situativ geboten sein, auch bei einer softwarebasierten Aufzeichnung mit weiteren technischen Geräten zu arbeiten: sei es, um die Aufnahme abzusichern (z.B. durch eine parallel filmende externe Kamera oder zumindest durch einen zusätzlichen Audio-Rekorder); sei es, um sie qualitativ aufzuwerten (z.B. durch künstliche Lichtquellen, akustische Verstärker für die Aufnahme der Audiospur u.ä.).

Kombinierte Bildschirm- und Personenvideographie ist - bei aller Vorteilhaftigkeit gerade für die medienpädagogische Forschung zum Internetumgang - auch mit gewissen Problemen und Limitationen behaftet. Ein zentraler Punkt ist das Reaktivitätsrisiko. Dieses lässt sich durch Rückgriff auf eine Software-Lösung zwar deutlich reduzieren, bleibt grundsätzlich aber in gewissem Masse bestehen. Wenngleich die Software unauffällig im Hintergrund läuft, so haftet der Gesamtkonstellation doch immer etwas Laborhaftes an. Die Forscherin oder der Forscher kann dem 
entgegenwirken, indem sie bzw. er sich mit möglichst minimalistischer Ausrüstung in das natürliche Umfeld der beforschten Personen begibt. Erfahrungsgemäss ist es in hohem Masse persönlichkeits- und aufgabenabhängig, inwieweit es den Probandinnen und Probanden gelingt, sich unter Erhebungsbedingungen derart in die jeweils interessierende Internetaktivität zu vertiefen, dass von authentischen Abläufen ausgegangen werden kann. Hilfreich ist es in jedem Fall, die Erhebungstechnik soweit möglich ausserhalb ihres Sichtfeldes zu platzieren, um unnötiger Irritation vorzubeugen. Ein weiterer Punkt ist, dass es sich bei Bildschirm- und Personenvideos nicht nur um hochgradig komplexe, sondern zudem um völlig unstrukturierte Forschungsdaten handelt. Dementsprechend aufwändig sind die Sichtungs- und Aufbereitungsprozesse, die der eigentlichen Auswertung noch vorgeschaltet werden müssen. ${ }^{6}$ Schliesslich ist auch zu bedenken, dass gängige Personenvideographie dort an ihre Grenzen stösst, wo Aspekte erforscht werden sollen, die entweder nicht mit blossem Auge bzw. normaler Kameraauflösung beobachtbar sind; oder die sich einer Beobachtung generell entziehen, weil sie innerlich verortet sind. In diesen Fällen bedarf es der Hinzunahme weiterer Erhebungsmethoden, die mittels spezieller Technik auch Beobachtungen höheren Auflösungsgrades ermöglichen (z.B. der Augenaktivität, s. u.); oder auch gezielt ausgewählter Verfahren, die Zugang zur Innenwelt der Probandinnen und Probanden eröffnen (vgl. Punkt (b)). Entscheidend ist gerade im Zusammenhang mit Videographie, sehr genau abzuwägen, welche zusätzliche Datensorte im jeweiligen Forschungskontext weiteren Erhebungsaufwand rechtfertigt.

\section{Eyetracking}

Als Eyetracking wird die videobasierte Registrierung des Blickverhaltens bezeichnet. Mittels hochauflösender Spezialkameras kann zum einen die Blickrichtung und somit das aktuell jeweils fokussierte Element eines betrachteten Bereichs erfasst werden. Zum anderen kann die Blickbewegung gemessen und nachgezeichnet werden, d.h. die Verweildauer des Blickes

6 Da Auswertungsaspekte nicht Thema dieses Beitrags sind, sei hier lediglich am Rande darauf hingewiesen, dass der für die Auswertung hinzukommende Aufwand hier noch nicht inbegriffen ist. Je nach Dynamik des videographisch erfassten Nutzer(innen)verhaltens kann die zu kalkulierende Auswertungszeit um ein Vielfaches höher liegen als die aufgezeichnete Zeit. 
auf einem bestimmten Element (Fixation) sowie die Blicksprünge zwischen verschiedenen Elementen (Sakkaden). Das videographierte Blickverhalten lässt sich softwaregestützt in unterschiedlicher Weise visualisieren. Beliebt sind in diesem Bereich vor allem Kartenvisualisierungen, bei denen im Hintergrund der Stimulus abgebildet ist (z.B. eine bestimmte Webseite) und eine zweite Bildebene zur Veranschaulichung des erfassten Blickverhaltens davorgelegt wird. Je nach Forschungsziel können die Blickverhaltensdaten entweder aggregiert oder als Verlauf dargestellt werden: Sogenannte Heatmaps veranschaulichen durch farbliche Kontraste die durchschnittliche Betrachtungshäufigkeit und -dauer von verschiedenen Elementen des Stimulusmaterials. Dagegen zeichnen sogenannte Gazeplots den gesamten Blickverlauf nach und geben somit Aufschluss darüber, in welcher Reihenfolge einzelne Stimuluselemente betrachtet werden. Neben Blickrichtung und Blickbewegungen können einige der heutzutage verfügbaren Eyetracking-Apparaturen auch noch weitere Bewegungsvorgänge am bzw. sogar im Auge registrieren, darunter Lidschlagfrequenz und Pupillenreflexe.

Die Erfassung des Blickverhaltens ist deshalb so aussagekräftig, weil das Auge sich stets aktiv und direkt auf den momentan verarbeiteten Reiz ausrichtet und insofern eine psychophysiologische Korrelation angenommen werden kann. Blickverhaltensdaten indizieren demnach, wieviel kognitiver Aufwand in die Verarbeitung des Stimulus bzw. seiner einzelnen Elemente investiert wird und wie sich die Verarbeitungsabfolge genau gestaltet. Je nachdem, wie viele Kennwerte der verwendete Eyetracker ermitteln kann, lassen sich ggf. auch Rückschüsse ziehen, welche Emotionen den 
kognitiven Verarbeitungsprozess begleiten. ${ }^{7}$ Der besondere Mehrwert von Eyetracking-Erhebungen liegt darin, dass man einen tiefgehenden Einblick in mentale Vorgänge erhält, ohne dabei jedoch den typischen Limitationen von Selbstauskunftsmethoden unterworfen zu sein. Im Gegensatz zu Letzteren liefert Eyetracking eine Art von Daten, die auch unbewusste sowie nicht verbalisiere Vorgänge abbilden. Diese Vorgänge werden zudem kontinuierlich und selbst über längere Zeitspannen hinweg so kleinschrittig abgebildet, wie es im Selbstbericht nicht möglich ist. Eyetracking-Daten weisen darüber hinaus ein vergleichsweise hohes Mass an Objektivität auf, da Blickverhalten sich nur bedingt steuern lässt.

Was die medienpädagogische Forschung zum Internetumgang betrifft, können Eyetracking-Daten sowohl für rezeptions- als auch für angebotszentrierte Untersuchungen aufschlussreich sein: also zum einen, es darum geht, informations- oder kommunikationsorientiertes Nutzer(innen)verhalten im Netz unter pädagogischen Gesichtspunkten zu erforschen (z.B. um auf dieser Basis geeignete Unterrichtskonzepte zu entwickeln, Selbstlernmaterial zu erstellen o.ä.); und zum anderen, wenn ein medienpädagogisch relevantes Internetangebot (beispielsweise ein onlinebasiertes Weiterbildungsangebot, eine Kindersuchmaschine o.ä.) hinsichtlich seiner konkreten Wirkung auf die Zielgruppe erforscht werden soll. Eyetracking-Daten können in diesem Zusammenhang hilfreich sein,

7 Eyetracking lässt sich dem Bereich psychophysiologischer Messmethoden zuordnen, d.h. letztlich soll über die Erfassung körperlicher Reaktionen auf mentale Vorgänge rückgeschlossen werden. Zwar bedarf es hochauflösender Spezialkameras, um Einzelheiten des Blickverhaltens sichtbar zu machen, dennoch werden beim Eyetracking überwiegend äusserlich sichtbare Körperreaktionen erfasst (im Gegensatz zu den im nächsten Abschnitt vorgestellten Methoden zur Messung innerer physiologischer Reaktionen, vgl. Punkt (b)). Freilich bewegen sich Messungen der Augenaktivität in einem Grenzbereich zwischen Körperoberfläche und Körperinnerem. Neben dem videobasierten Zugang mittels Eyetracking gibt es noch ein anderes Verfahren zur Erfassung von Augenaktivität, das sich sehr viel klarer der Messung innerer Körperreaktionen zuordnen lässt. Bei der sogenannten Elektrookulographie (EOG) wird mit Elektroden gearbeitet, um äusserlich nicht sichtbare Potenzialschwankungen im Augen zu erfassen und daraus auf Blickrichtungswechsel rückzuschliessen. Aufgrund der vergleichsweise geringeren Präzision ist die EOGMethode für die Bearbeitung medienpsychologischer und -pädagogischer Forschungsfragen aber weniger zu empfehlen (Kempter und Bente 2004, 284) und wird hier daher nicht weiter ausgeführt. 
um Optimierungsbedarfe zu erkennen und den Nutzerinnen und Nutzern z.B. eine schnellere und einfachere Orientierung oder eine intuitivere $\mathrm{Na}$ vigation zu ermöglichen, um stärkere motivationale Anreize zur Beschäftigung mit bestimmten Seitenbereichen zu schaffen usw. Unbedingt zu berücksichtigen ist, dass Augenaktivität nur unter bestimmten Bedingungen als verlässlicher Indikator für die Zu-/Abwendung von Aufmerksamkeit und Interesse bzw. für einen mehr oder minder grossen Verarbeitungsaufwand und ggf. für begleitende Emotionen interpretiert werden kann: Dafür muss gewährleistet sein, dass die Probandinnen und Probanden im betreffenden Kontext in ein zielgerichtetes Schauen involviert sind (und nicht z.B. in ein gedankenverlorenes (Vor-sich-hin-Blicken), bei dem der Zielpunkt an sich gar nicht interessiert) (vgl. für Einzelheiten zu verschiedenen Arten des Schauens: Bente 2004, 306f.). Zu empfehlen ist daher, für die Analyse von Rezeptionsverhalten wie auch für Wirkungsanalysen zu bestimmten Webangeboten, ausschliesslich Blickverhaltensdaten von Personen zu nutzen, die während des Eyetrackings eine entsprechend konzipierte Aufgabe zur Auswertung visueller Informationen bearbeitet haben (ebd., 310). Nur dann lassen sich aus der Anzahl und Dauer von Fixationen, aus der Grösse und Richtung von Sakkaden, aus Lidschlagfrequenz und Pupillenreaktionen - vor allem in der Zusammenschau mehrerer diesbezüglicher Kennwerte - interessante Rückschlüsse ziehen und beispielsweise konzentriert-ausgeglichene Blickmuster von solchen unterscheiden, die eine unaufmerksame Rezeption, Orientierungsverlust, Verständnisschwierigkeiten oder sonstige Stressoren erkennen lassen. Exemplarisch sei auf eine Publikation verwiesen, in der die Entwicklung und Testung eines adaptiven, auf individuelle Lerner(innen)bedürfnisse reagierenden Online-Lernraums beschrieben wird. Die Adaptivität des Systems wird hier dadurch sichergestellt, dass mittels Eyetracking-Daten der Nutzerinnen und Nutzer auf deren Schwierigkeiten bzw. Unterstützungsbedarfe rückgeschlossen wird (Leber und Skuballa 2014).

Um eine Eyetracking-Erhebung durchführen zu können, bedarf es einer entsprechenden technischen Ausstattung. Die Anschaffung hochwertiger Blickbewegungskameras und zugehöriger Tracking-Software ist kostenintensiv, wobei es hier je nach Gerätetyp eine grosse Spannbreite gibt und inzwischen auch deutlich günstigere Preisangebote zu finden 
sind als noch vor einigen Jahren. Tendenziell dürften im Zuge des technischen Fortschritts insbesondere Wearable-Eyetracker, die in Aussehen und Funktionsweise VR-Brillen ähneln, erschwinglicher werden. Um die Kosten überschaubar zu halten, kann es je nach Gerätetyp und Nutzungsdauer eine gute Alternative sein, von einer Anschaffung abzusehen und Eyetracking-Apparaturen jeweils bedarfsbezogen und auf aktuellstem Stand der Technik zu mieten. Des Weiteren ist Forscherinnen und Forschern zu empfehlen, im Vorhinein einen gewissen zeitlichen Aufwand einplanen: zunächst um sich einen Überblick über die Vor- und Nachteile verschiedener technischer Lösungen zu verschaffen; und um sich anschliessend mit der Funktionsweise des jeweiligen Geräts vertraut zu machen, sodass die verschiedenen Funktionen im Sinne des jeweiligen Erkenntnisinteresses optimal ausgeschöpft werden können.

Welche Limitationen im Einzelnen zu berücksichtigen sind, hängt vom Gerätetyp ab (vgl. für Details: Pelz 2019): Die besonders messgenauen Tower-Systeme mit Kopffixierung nehmen den Untersuchungspersonen jegliche Bewegungsfreiheit. So bleibt die Laborsituation dauerpräsent, was ein erhöhtes Reaktivitätsrisiko bedingt. Bei Remote-Systemen hingegen besteht keinerlei Körperkontakt. Die gesamte Situation gestaltet sich dadurch deutlich natürlicher und weniger reaktiv. Zu bedenken ist allerdings, dass Kopfbewegungsartefakte mittels Kalibrierung aus den Daten herausgefiltert werden müssen und Einbussen hinsichtlich Messgenauigkeit und Bildauflösung nach wie vor in Kauf zu nehmen sind. Da bei stationären Geräten also keine nennenswerte Lokomotion möglich ist, beschränkt sich ihr Einsatz generell auf die Erforschung von sitzend ausgeübten bzw. sehr bewegungsarmen Tätigkeiten. Mobile Eyetracker in Form von Headsets oder Wearables, die sowohl Bewegungsfreiheit als auch eine hohe Messqualität erlauben, sind wiederum mit dem Nachteil verbunden, dass das Gerät am Kopf der untersuchten Person befestigt ist und zumindest ein Fremdkörpergefühl auslösen dürfte, verbunden mit entsprechenden Einbussen an ökologischer Validität bzw. erhöhtem Reaktivitätsrisiko. Unabhängig von der Art der Messapparatur ist schliesslich zu bedenken, dass Augenbewegungen zwar als starker physischer Indikator für mentale Vorgänge gelten können, Letztere aber auf diese Weise dennoch nicht direkt zugänglich sind, sondern unter Einbezug von Kontextwissen erschlossen 
werden müssen. Hilfreich ist, möglichst viele okulometrische Kennwerte parallel zu erfassen und bei der Interpretation aufeinander zu beziehen. Gerade für den Einsatz innerhalb der medienpädagogischen Forschung ist zu empfehlen, Eyetracking als Komplementärmethode anzusehen und stets in Kombination mit anderen Beobachtungs-, Mess- und/oder Verbalisierungsmethoden einzusetzen.

(b) Erfassung innerlicher Prozesse des Internetumgangs einzelner Nutzerinnen und Nutzer

Zu den innerlichen Prozessen zählen all solche, die in der Innenwelt der Probandin bzw. des Probanden stattfinden. Gemeint sind zum einen bestimmte innere Körperreaktionen, die sich mittels biometrischer Messverfahren erfassen und als Indikatoren für mentale Vorgänge deuten lassen. Zum anderen sind aber auch diese mentalen Vorgänge selbst gemeint, d.h. Kognitionen, Emotionen und motivational-volitionalen Regungen, die im Umgang mit einer konkreten Anforderungssituation innerlich ablaufen und als solche zwar nicht direkt zugänglich sind, aber von Probandinnen und Probanden zumindest teilweise prozessbegleitend verbalisiert werden können. Innere Prozesse genauer zu untersuchen - sei es nun auf Basis psychophysiologischer Indikatoren und/oder proband(inn)enseitiger Verbalisierungen - scheint gerade für die Erforschung von Umgangsweisen mit Internettechnologien aufschlussreich, weil Internetnutzerinnen und -nutzer mit besonders intensiven, multi- und hypermedialen Stimuli konfrontiert werden. Dadurch werden entsprechend komplexe kognitive und affektive Verarbeitungsvorgänge ausgelöst, die massgeblich für alle äusserlich ablaufenden Prozesse sind.

Innerliche Aspekte des Umgangs mit Internettechnologien entziehen sich grundsätzlich der unmittelbaren Fremdbeobachtung und Protokollierung bzw. Aufzeichnung durch die oder den Forschenden. Möglich ist hingegen eine mittelbare Beobachtung, d.h. zunächst müssen spezielle biometrische und/oder kognitionspsychologische Verfahren vorgeschaltet werden, die Innerliches - also innerlich ablaufende Körperreaktionen und mentale Vorgänge - in Werte bzw. Worte fassen und dadurch «beobachtbar> machen. Erst durch diesen Zwischenschritt entstehen also auswertbare Forschungsdaten. Für die medienpädagogische Forschung zum 
Internetumgang kann sowohl der biometrische als auch der kognitionspsychologische Zugang aufschlussreich sein:

\section{Messung innerer physiologischer Reaktionen}

$\mathrm{Zu}$ den relevanten Messgrössen bzw. Messverfahren im Bereich innerer Körperfunktionen zählen insbesondere: die kardiovaskuläre Aktivität (messbar mittels EKG, peripherer Pulsmessung und Blutdruckmessgeräten); die Atmungsaktivität (messbar mittels MPG); die elektrodermale Aktivität (messbar mittels EDG); die muskuläre Aktivität (messbar mittels EMS); und nicht zuletzt die Endhirnaktivität (messbar mittels EEG und ggf. MRT). ${ }^{8}$ Die Durchführung von Messungen in all diesen Bereichen basiert auf der Annahme, dass messbare Schwankungen der Herzschlagfrequenz und des Blutdrucks, der Atemfrequenz, des Hautleitwertes, des Muskeltonus und der Hirnströme mit mentalen Vorgängen korrespondieren und diese somit indizieren. Dabei wird in Anlehnung an allgemeine Aktivierungstheorien angenommen, dass ein hohes Mass an physiologischer Aktivierung auch ein hohes Mass an emotionalem und/oder kognitivem Involvement bedeutet. Hohe Messwerte während einer Internetsitzung können also beispielsweise auf besondere Stressfaktoren im Umgang mit einer Webanwendung hinweisen, selektive Aufmerksamkeitsprozesse bei der Rezeption komplexer Informationsreize verdeutlichen, etwaige Motivationsschwankungen im Umgang mit einer Online-Lernumgebung anzeigen usw. Psychophysiologische Messungen bieten den grossen Vorteil, gewisse Anhaltspunkte auch zu solchen mentalen Zuständen und Vorgängen zu liefern, die in proband(inn)enseitigen Verbalisierungen nicht enthalten sind. Dies betrifft zum einen hochautomatisierte Vorgänge, die sich generell der Bewusstseinsfähigkeit entziehen; und zum anderen Vorgänge, die sich in Worten nicht angemessen ausdrücken lassen. Vorteilhaft erweist sich in diesem Zusammenhang auch, dass die Messung von Biosignalen objektivere Vergleiche zwischen Untersuchungspersonen ermöglicht

8 Freilich sind diese überwiegend innerlich stattfindenden physiologischen Reaktionen stellenweise an der Körperoberfläche und somit in gewissem Masse auch ohne die jeweilige Messtechnik feststellbar, z.B. durch hörbar beschleunigte Atmung, Erröten der Haut, eine sichtlich verkrampfte Körperhaltung und dergleichen. Für die Erstellung detailgenauer Verlaufskurven zu wissenschaftlichen Zwecken bedarf es jedoch zuverlässiger Messungen mittels entsprechender Apparaturen. 
Verbalisierungsmethoden: So spielt es bei den genannten Messverfahren keine Rolle, dass die Fähigkeit zur Selbstbeobachtung innerlicher Zustände und Vorgänge individuell ganz unterschiedlich ausgeprägt sein kann. Auch stellen unterschiedliche Verbalisierungsfähigkeiten kein Problem dar; ebenso wenig die Tatsache, dass verschiedene Personen vergleichbare Bewusstseinsinhalte womöglich sehr unterschiedlich wahrnehmen und umschreiben. Des Weiteren sind psychophysiologische Messungen nicht anfällig für subjektive Darstellungstendenzen (Ausrichtung an sozialer Erwünschtheit etc.), weil die betreffenden Körperreaktionen sehr schnell und unwillkürlich ausgelöst werden und daher kaum kontrollierbar sind. Ein weiterer Vorteil besteht darin, dass die Erfassung psychophysiologischer Indikatoren kontinuierliche Verlaufsanalysen auch über längere Zeiträume hinweg ermöglicht, weil die Aussagekraft der Daten nicht durch (unterschiedlich schnell eintretende) Ermüdungseffekte der Probandinnen und Probanden beeinträchtigt wird. Nicht zuletzt weisen psychophysiologische Messungen den Vorteil einer vergleichsweise geringen Reaktivität auf, weil heutige Messapparaturen sehr unauffällig einsetzbar sind und in aller Regel nach kurzer Gewöhnungszeit von den beforschten Personen vergessen werden. Insgesamt ist von einem hohen Erkenntnispotenzial gerade auch für die medienpädagogische Forschung auszugehen, wo die Messung innerer Körperreaktionen allerdings bislang kaum fruchtbar gemacht wird (Kempter und Bente 2004, 274). Besonders vielversprechend scheinen Entwicklungen im Bereich Mobile Research, wodurch zunehmend flexible und unauffällige Messungen mittels Smart Wearables möglich werden. Beispielhaft sei auf eine frühe Studie zur Erfassung emotionaler Belastung bei der Rezeption unterschiedlicher Videostimuli verwiesen, in der unter Verwendung eines Smartbands im häuslichen Umfeld gearbeitet werden konnte (Papastefanou 2009, 461f.)

Insbesondere wenn mobile Geräten zum Einsatz kommen, lassen sich psychophysiologische Messungen inzwischen ohne grossen Zeit- und Kostenaufwand umsetzen. Dafür werden Elektroden an verschiedenen Stellen der Körperoberfläche aufgeklebt, die die empfangenen Rohsignale an ein spezielles Gerät zur Umwandlung und Speicherung weiterleiten. Moderne Messapparaturen sind klein, handlich und portabel einsetzbar, sodass die oder der Forschende die Probandinnen und Probanden damit ins normale 
Alltagsgeschehen schicken kann und während der Messung nicht durchgängig zugegen sein muss. Die Bedienung ist inzwischen recht einfach und bedarf keiner langen Vorbereitung. Entsprechende Untersuchungen im Labor sind nicht nur deutlich zeit- und kostenintensiver, sondern setzen auch umfangreichere erhebungstechnische Bedienfertigkeiten seitens der Forscherin oder des Forschers voraus.

$\mathrm{Zu}$ bedenken ist eine limitierte Aussagekraft psychophysiologischer Messungen dahingehend, dass es sich bei den ermittelten Kennwerten zunächst einmal um unspezifische Indikatoren mentaler Aktivität handelt, d.h. in den Werten spiegelt sich lediglich die Intensität kognitiver und affektiver Vorgänge, nicht jedoch deren Erlebensqualität. Bezogen auf Situationen des Internetumgangs bedeutet dies, dass sich aus hohen Aktivitätsmassen allein nicht eindeutig ablesen lässt, ob die Untersuchungsperson sich in dem Moment z.B. über eine langsame Datenverbindung aufregt, mit grosser Begeisterung an einem Online-Spiel teilnimmt oder sich intensiv mit den Informationen auf einer Webseite auseinandersetzt. Bislang konnten nur einige wenige Reaktionsmuster klar voneinander abgegrenzt und kontextunabhängig mit einer spezifischen Erlebensqualität in Verbindung gebracht werden, darunter z.B. eine typische Orientierungsreaktion, eine Defensiv- und eine Schreckreaktion (vgl. ausführlicher: Kempter und Bente 2004, 281). Unabdingbar für deren Identifizierung ist es, stets Breitbandmessungen zu mehreren Indikatoren durchzuführen, da isolierte Werte nicht verlässlich interpretierbar sind. Darüber hinaus gilt, dass psychophysiologische Messungen vor allem als Komplementärmethode eingesetzt werden sollten. Im Verbund mit beobachtenden Verfahren (vgl. Punkt (a), Lautem Denken (s.u.) und/oder Befragungsverfahren ${ }^{9}$ können sie Forschungserkenntnisse absichern und vertiefen.

\section{Aufzeichnung von Lautem Denken}

Anders als die Messung physiologischer Reaktionen liefert die Methode des Lauten Denkens (think aloud) inhaltlich spezifische Daten über mentale Vorgänge während des Umgangs mit dem Internet. Beim Lauten Denken soll die Untersuchungsperson parallel zu ihren internetbezogenen

9 Gemäss der einleitend vorgeschlagenen Systematisierung sind Befragungen dem Methodenbereich (B) zuzuordnen und werden daher im Rahmen dieses Beitrags nicht thematisiert (vgl. Punkt 1). 
Aktivitäten fortwährend laut aussprechen, welche Gedanken ihr gerade durch den Kopf gehen und was sie dabei empfindet. Das Verfahren ist kognitionstheoretisch fundiert und wurde in umfangreichen methodenprüfenden Studien validiert (Ericsson und Simon 1993). Sofern bestimmte methodische Prämissen erfüllt sind, lassen sich valide Daten gewinnen, die tiefgehende Einblicke in die Wahrnehmungs-, Verarbeitungs- und Entscheidungsprozesse einzelner Personen gewähren. Als entscheidend hat sich herausgestellt, dass die untersuchte Person all ihre handlungsbegleitend aufkommenden Gedanken und Empfindungen sofort und völlig unreflektiert vor sich hinspricht, ohne ihre Äusserungen an die Forscherin bzw. den Forscher zu adressieren. Anderenfalls, so konnte vielfach nachgewiesen werden, ist mit erheblichen Validitätseinbussen zu rechnen: Sobald Äusserungen explizit an eine andere anwesende Person, hier also an die Forscherin oder den Forscher, gerichtet werden, wechseln Probandinnen und Probanden vom Modus des Lauten Denkens in einen Modus des Beschreibens und Erklärens. Die mental ablaufenden Prozesse werden dann nicht mehr in Reinform wiedergegeben, sondern unwillkürlich für das Gegenüber (aufbereitet), d.h. im Hinblick auf Nachvollziehbarkeit überdacht und restrukturiert, ggf. optimiert und ausformuliert. Infolgedessen entsteht eine völlig andersartige Datensorte. Nur solange der Akt des Aufbereitens ausbleibt, werden tatsächlich Daten im Sinne Lauten Denkens generiert, die sich als Abbild mentaler Prozesse interpretieren lassen. ${ }^{10}$ Problematisch wird es, wenn Fehlettikettierungen von Selbstberichten als vermeintlich Lautes Denken auch damit einhergehen, dass die generierte Datensorte epistemologisch falsch eingeordnet wird - was selbst in ansonsten gut konzipierten Studien nicht selten der Fall ist. Derart

10 Auch beschreibende und erklärende (und somit (aufbereitete)) Verbalisierungen erzeugen freilich aussagekräftige Daten. Zu berücksichtigen ist allerdings, dass mentale Vorgänge bei solchen Verbalisierungen nicht einfach unbereinigt ausgesprochen werden, sondern vielmehr über diese Vorgänge gesprochen wird. Bei beschreibenden und erklärenden Verbalisierungen handelt es sich also anders als beim Lauten Denken nicht um eine kognitionspsychologische Methode, die Zugang zu faktisch ablaufenden innerlichen Prozessen eröffnet, sondern um eine Selbstberichtsmethode. Selbstberichtsmethoden eröffnen Zugang zu subjektiven Sichtweisen und Wissensbeständen. Im Sinne der diesem Beitrag zugrunde liegenden Systematisierung fällt Lautes Denken in den Methodenbereich $(A)$, wohingegen Selbstberichte dem hier nicht weiter zu vertiefenden Methodenbereich (B) zuzuordnen sind. 
elementare Kategorienfehler ziehen Fehlinterpretationen nach sich und führen letztlich zu nicht verwertbaren Untersuchungsergebnissen.

Methodisch sauber evoziertes Lautes Denken vereint mehrere Vorteile in sich: Bedingt durch die Unreflektiertheit der Äusserungen ist von einem minimierten Reaktivitätsrisiko auszugehen. Der Einsatz der Methode induziert per se keine strukturellen Änderungen des natürlichen Vorgehens: ganz einfach deshalb, weil Letzteres beim Lauten Denken nicht zusätzlich reflektiert und somit auch nicht eher als optimierungsbedürftig erkannt wird als unter natürlichen Bedingungen. Ein anderer positiver Nebeneffekt der ausbleibenden Reflexion ist die Minimierung subjektiver Darstellungstendenzen, weil die Probandin bzw. der Proband keine Gelegenheit hat, darüber nachzudenken, ob das Ausgesprochene einen guten oder schlechten Eindruck hinterlässt. Aufgrund der Handlungsparallelität der Äusserungen spielen ausserdem Erinnerungslücken und -fehler beim Lauten Denken praktisch keine Rolle. Ein weiterer Mehrwert ist schliesslich auch darin zu sehen, dass sich in Lautem Denken die subjektiven Relevanzsetzungen der jeweiligen Person sehr deutlich widerspiegeln, weil die Forscherin bzw. der Forscher zwecks Sicherung der Datenvalidität bestenfalls kaum interveniert. Als Beispiel für eine methodisch einwandfrei realisierte Datenerhebung sei auf Richter u. a. (2005) verwiesen, die u. a. anhand von Lautem Denken den Einsatz kognitiver und metakognitiver Lernstrategien in Print- und Hypertextumgebungen untersuchen. Gerhardts (in Vorb.) arbeitet ebenfalls mit Lautem Denken, wobei die Daten hier genutzt werden, um Kompetenzkomponenten im Bereich Internetrecherche zu ermitteln (vgl. auch Punkt 3).

Was den Aufwand und das erforderlichen Know-how betrifft, ist die Methode des Lauten Denkens recht voraussetzungsvoll. So ist unbedingt zu empfehlen, sich als Forscherin bzw. Forscher zuvor genau mit der kognitionstheoretischen Fundierung und dem methodenkritischen Diskurs rund um dieses Verfahren auseinanderzusetzen. Nur so versteht man die weitreichende Bedeutung der daraus erwachsenen Anwendungsleitlinien und kann in der Erhebungssituation entsprechend souverän reagieren. Auch sollte Zeit eingeplant werden, um das Laute Denken mit den Probandinnen und Probanden im Vorhinein der Erhebung zu üben und dabei verständlich zu machen, welche Art von Verbalisierungen von ihnen erwartet 
wird. Erfahrungsgemäss hat nur eine Minderheit der Untersuchungspersonen anhaltende Schwierigkeiten mit Lautem Denken.

Limitationen bestehen dahingehend, dass der Anwendungsbereich Lauten Denkens grundsätzlich auf solche Prozesse beschränkt ist, die sich aus Inhalten des Arbeitsgedächtnisses rekonstruieren lassen (d.h. dass die Methode sich zur Untersuchung von Wahrnehmungs-, Verarbeitungs- und Entscheidungsprozessen im Zuge von Internetsitzungen in besonderem Masse eignet, für andere Forschungsbereiche wiederum von vornherein auszuschliessen ist). Zu bedenken ist des Weiteren, dass sich bei der Wiedergabe mentaler Vorgängen mittels Lautem Denken prinzipiell niemals Vollständigkeit erreichen lässt. Nicht alles, was mental abläuft, ist in demselben hohen Tempo auch aussprechbar, d.h. es kommt immer wieder zu Raffungen und Auslassungen. Darüber hinaus fehlen in Laut Denk-Protokollen, wie in allen Arten von Verbaldaten, auch jegliche Hinweise zu nicht bewusstseinsfähigen und/oder nicht verbalisierbaren Aspekten. Unter dem Strich ist dennoch von einem enormen Erkenntnispotenzial des Lauten Denkens auszugehen. Dies gilt umso mehr, wenn die Methode nicht isoliert eingesetzt wird, sondern im Zusammenhang mit einer Beobachtungsstudie. Speziell im Bereich der medienpädagogischen Internetforschung empfiehlt sich hier oftmals eine kombinierte Personen- und Bildschirmvideographie (vgl. Punkt (a)), die ergänzende Anhaltspunkte für die unausgesprochenen Aspekte der Mensch-Computer-Interaktion liefert.

\subsubsection{Erfassung von Produkten des Internetumgangs einzelner Nutzerinnen und Nutzer}

Produktdaten werden erhoben, nachdem die für das jeweils interessierende Umgangsphänomen charakteristischen Vorgänge bereits abgelaufen sind und zu bestimmten (Zwischen-)Ergebnissen geführt haben. Die Erhebung von Produktdaten ermöglicht gleichsam eine indirekte (soll heissen: zeitversetzte) Form wissenschaftlicher Beobachtung und lässt sich eingruppieren als 


\section{Dokumentenanalyse mit Ausrichtung auf Prozessrekonstruktion}

Genuine Dokumentenanalysen - im Sinne der gezielten Suche und Systematisierung von forschungsrelevanten, aber nicht forschungsgenerierten Dokumenten - stellen eine separate Methode der empirischen Datenerhebung dar (und keine Auswertungsmethode ${ }^{11}$ ). Bei Dokumentenanalysen geht es um das Sammeln von natürlicherweise entstandenen, unabhängig von der jeweiligen Forschung erzeugten Dokumenten bzw. Artefakten unterschiedlichster Art. Dabei kann es sich um - offline und/oder online zugängliche - schriftsprachliche, visuelle, audiovisuelle, multi- oder hypermediale Produkte handeln. Grundlegend ist der Gedanke, dass sich in bestimmten, begründet auszuwählenden Dokumenten bzw. Artefakten das im jeweiligen Forschungskontext interessierende Erleben und Verhalten von Menschen manifestiert, das auf dieser Basis dann aus der Retrospektive analysiert werden kann. Alle Arten von Dokumenten bzw. Artefakten lassen sich grundsätzlich sowohl für die Rekonstruktion von Sichtweisen und Wissensbeständen nutzen als auch für die Rekonstruktion faktischer Handlungsprozesse, die an der Entstehung bzw. Erzeugung des jeweiligen Produkts beteiligt gewesen sein müssen. Für die Erforschung des faktischen Internetumgangs einzelner Nutzerinnen und Nutzer ist also die zweite, auf Prozessrekonstruktion ausgerichtete Variante interessant. ${ }^{12}$ Die Sammelaktivität der oder des Forschenden zielt dementsprechend auf Dokumente, die aus der jeweils interessierenden Art von Internetumgang hervorgegangen sind und daher - zumindest bedingt - auf einige vorausgegangene Prozesse rückschliessen lassen. So können beispielsweise Recherchenotizen hilfreich sein, um bestimmte Aktivitäten zu rekonstruieren, die im Rahmen der zugehörigen Internetrecherche stattgefunden haben müssen. Ein weiteres Beispiel wären persönliche Web-Auftritte wie Social Media Profile, eigene Weblogs u.ä., die sich einer einzelnen Person zuordnen lassen, nachweislich von dieser selbst gestaltet werden und

11 Per Dokumentenanalyse erhobene Forschungsdaten lassen sich je nach Forschungsinteresse anschliessend mit allen gängigen qualitativen Analyseverfahren auswerten bzw. nach einer entsprechenden Aufbereitung auch mittels statistischer Verfahren (Döring und Bortz 2016, 535).

12 Dokumentenanalysen mit Ausrichtung auf die Rekonstruktion von Sichtweisen und Wissensbeständen wären gemäss der hier gewählten Systematisierung dem Methodenbereich (B) zuzuordnen und sind daher an dieser Stelle nicht zu vertiefen. 
somit Rückschlüsse auf bereits eingeflossene gestalterische Aktivitäten erlauben. Der grosse Vorteil von Datenerhebungen mittels Dokumentenanalyse liegt in der Non-Reaktivität des Verfahrens. Im Rahmen medienpädagogischer Forschungszusammenhänge sind Web-Dokumentenanalysen noch rar (Bettinger 2018), insbesondere Analysen mit expliziter Ausrichtung auf die Rekonstruktion des Internetumgangs einzelner Nutzerinnen und Nutzer. Angaben zum zeitlichen und finanziellen Aufwand sowie zum erforderlichen Know-how sind pauschal kaum möglich. Zeit und Kosten der Beschaffung sowie die dafür erforderlichen Vorkenntnisse hängen unmittelbar von der Art des jeweiligen Dokuments bzw. Artefakts ab und können mitunter stark variieren. Allgemein gelten Dokumentenanalysen aber als eine vergleichsweise forschungsökonomische Form der Datenerhebung. $\mathrm{Zu}$ bedenken sind Limitationen von Dokumentenanalysen dahingehend, dass oftmals nur wenig über den Entstehungs-/Herstellungskontext eines Dokuments bekannt ist, was das Risiko von Fehlinterpretationen erhöht. Des Weiteren ist bei vorgefundenen Dokumenten der Aussagegehalt in Bezug auf die eigene Forschungsfrage ungewiss und durch die oder den Forschenden nicht beeinflussbar. Die Kombination mit prozesserfassenden Methoden (vgl. Punkt 2.2.1) ist daher in jedem Fall zu empfehlen.

\section{Anschauungsbeispiele}

Nachfolgend werden zwei medienpädagogische Forschungsbeispiele zur Untersuchung von Umgangsweisen mit dem Internet vorgestellt, in deren Zentrum - erhebungsmethodisch gesehen - jeweils die Durchführung einer kombinierten Bildschirm- und Personenvideographie steht. Durch die unmittelbare Gegenüberstellung der beiden Beispiele soll anschaulich werden, wie sich mittels gezielter Methodentriangulation ein gegenstandsgerechtes Erhebungsdesign realisieren lässt. 


\section{Beispiel 1: Modellierung von Internetrecherchekompetenz für (schul-)didaktische Anwendungen}

Ein grundlegender Schritt innerhalb des laufenden Dissertationsvorhabens der Verfasserin ${ }^{13}$ bestand darin, alle wesentlichen Komponenten des Konstrukts Internetrecherchekompetenz zu identifizieren. Um auch etwaige unvorhergesehene Kompetenzaspekte ermitteln zu können, wurde unter anderem ein induktiver Zugang realisiert. Dafür wurden kombinierte Bildschirm- und Personenvideographien von Schülerinnen und Schülern der Sekundarstufe I in realitätsnahen schulischen Recherchesituationen durchgeführt. Parallel kam die Erhebungsmethode des Lauten Denkens zum Einsatz. Ergänzend wurden zudem qualitative Anschlussinterviews geführt, in die eine Phase mit videostimulierten Rückblicken auf den eigenen Rechercheprozess integriert war (Gerhardts in Vorb.). Abbildung 1 zeigt einen exemplarischen Screenshot aus dem recherchebegleitend generierten Datenmaterial.

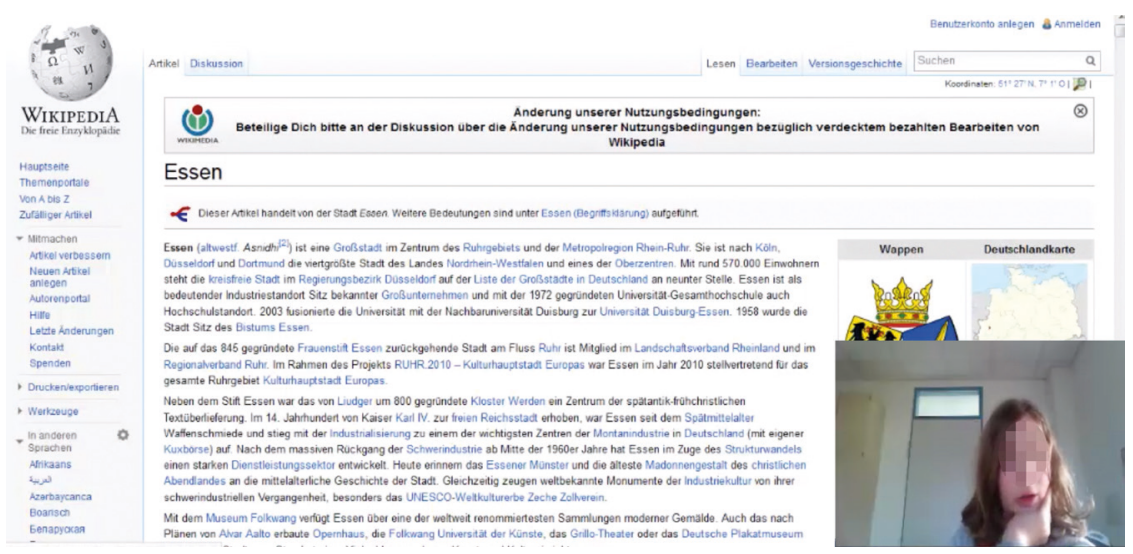

Abb. 1.: Schülerinnen- bzw. Schülerrecherche im schulischen Kontext.

13 Die Dissertation wird von Dorothee M. Meister als Erstgutachterin an der Universität Paderborn betreut. 


\section{Beispiel 2: Untersuchung elterlichen Unterstützungsverhal- tens bei der informationsorientierten Internetnutzung}

Um die Rolle von Eltern im Kontext der informationsorientierten Internetnutzung ihrer Kinder untersuchen zu können, ${ }^{14}$ wurde in Anlehnung an das im o. g. Dissertationsvorhaben bereits erprobte Erhebungsdesign die folgende Methodentriangulation realisiert: Mittels kombinierter Personen- und Bildschirmvideographie wurden dyadische Kind-und-ElternteilRecherchen aufgezeichnet. Im Sinne einer Constructive Interaction waren die Probandinnen und Probanden angewiesen, während der gesamten gemeinsamen Recherche möglichst natürlich miteinander zu kommunizieren. ${ }^{15}$ Auch hier wurde ergänzend sowohl mit dem Kind als auch mit dem Elternteil jeweils ein qualitatives Anschlussinterview geführt, in das eine Phase mit videostimulierten Rückblicken auf den Rechercheprozess integriert war. Abbildung 2 zeigt einen exemplarischen Screenshot aus dem recherchebegleitend generierten Datenmaterial.

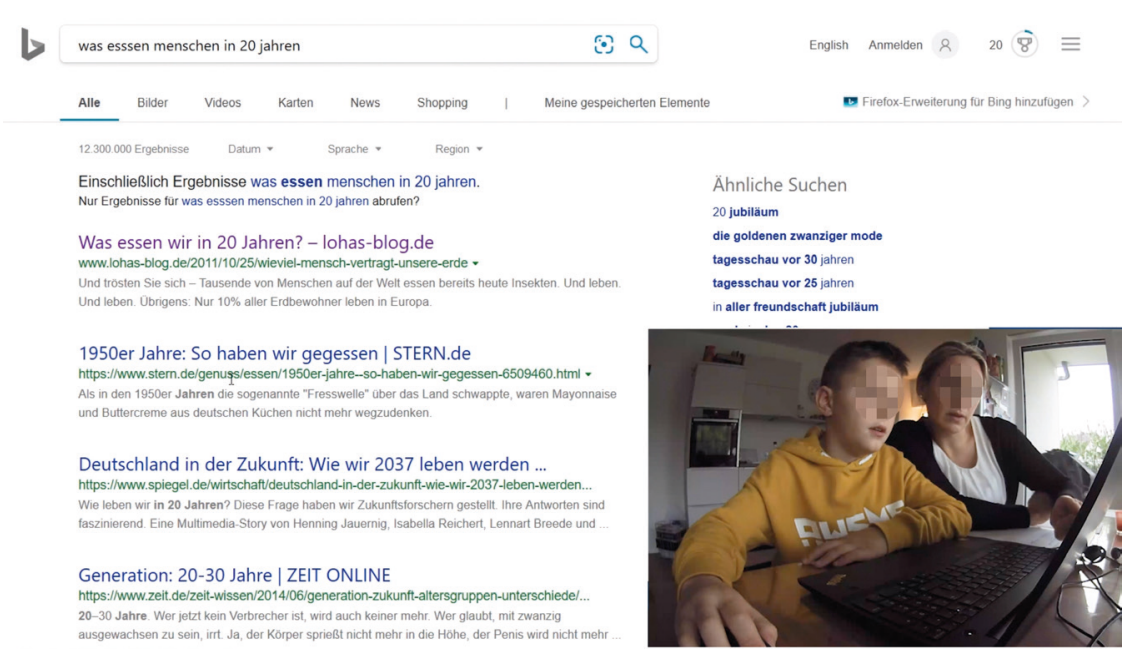

Abb. 2.: Kind-und-Elternteil-Recherche im häuslichen Kontext.

14 Die Untersuchung findet im Rahmen des von Dorothee M. Meister geleiteten Teilprojekts innerhalb des BMBF-geförderten Forschungsverbundes DigHomE (Digital Home Learning Environment) statt (https://digi-ebf.de/dighome).

15 Die Methode der Constructive Interaction generiert Sichtweisen- und Wissensäusserungen und fällt im Sinne der hier gewählten Systematisierung somit in den Methodenbereich (B). Auf weitere Ausführungen wird dementsprechend an dieser Stelle verzichtet. 


\section{Ausblick}

Angesichts der grossen Entwicklungsdynamik von Internettechnologien können Aussagen zu künftigen technischen Möglichkeiten immer nur unter Vorbehalt getroffen werden. Einige Trends von übergeordneter Bedeutung - auch und insbesondere für künftige erhebungsmethodische Entwicklungen im Bereich der internetbezogenen medienpädagogischen Forschung - seien zumindest stichpunktartig angedeutet:

- Mobile Research: neue erhebungsmethodische Spielräume, vor allem durch Wearable-basierte Verfahren der Datenerhebung;

- Emotional Computing: zunehmende Bedeutung psychophysiologischer Datenerhebungsverfahren;

- Big Data: Weiterentwicklungen bei der Aufbereitung automatisch generierter riesiger Datensätze, speziell auch für die Zwecke (medien-) pädagogische Forschung;

- Remote Research: neue erhebungsmethodische Spielräume durch onlinebasierte Fernzugriffe, angeregt durch forschungspraktische Notwendigkeiten in Zeiten des pandemiebedingten Lockdowns

Die medienpädagogische Forschung zum Internetumgang kann von Entwicklungen in all diesen Bereichen nur profitieren.

Für wichtige Anmerkungen zum Manuskript danke ich meinem Peer Coach Dr. Christina Watson sowie meiner Kollegin Jeannine Teichert. Auch habe ich einige Ideen für diesen Text aus umfangreichen Diskussionen mit meinem technikbegeisterten Kollegen Florian Müller gewonnen, dem ich an dieser Stelle ebenfalls danken möchte. 


\section{Literatur}

Bente, Gary. 2004. «Erfassung und Analyse des Blickverhaltens». In Lehrbuch der Medienpsychologie, herausgegeben von Roland Mangold, Peter Vorderer, und Gary Bente, 297-342. Göttingen u.a.: Hogrefe.

Bettinger, Patrick. 2018. Praxeologische Medienbildung. Theoretische und empirische Perspektiven auf sozio-mediale Habitustransformationen. Wiesbaden: Springer VS.

Carstensen, Tanja, und Gabriele Winker. 2012. «Intersektionalität in der Internetforschung». M\&K 1: 1-23.

Döring, Nicola, und Jürgen Bortz. 2016. Forschungsmethoden und Evaluation in den Sozial- und Humanwissenschaften. Springer-Lehrbuch. Berlin, Heidelberg: Springer Berlin Heidelberg. https://doi.org/10.1007/978-3-642-41089-5.

Ducheneaut, Nicolas, und Robert J. Moore. 2005. «More than Just 'XP': Learning Social Skills in Massively Multiplayer Online Games». Interactive Technology and Smart Education 2 (2): 89-100. https://doi.org/10.1108/17415650580000035.

Ericsson, K. A., und H. A. Simon. 1993. Protocol analysis: Verbal reports as data. 2nd, rev. ed. Aufl. Cambridge, MA: MIT Press.

Feil, Christine, Christoph Gieger, und Alexander Grobbin. 2013. «Informationsverhalten von Kindern im Internet: Eine empirische Studie zur Nutzung von Suchmaschinen». Diskurs Kindheits- und Jugendforschung 2: 223-29.

Flick, Uwe. 2011. Triangulation. Eine Einführung. 2. Aufl. Wiesbaden: VS Verlag.

Gerhardts, Lara. in Vorb. Recherchieren im Internet - Konstruktion eines Kompetenzstrukturmodells für (schul-)didaktische Anwendungen. Paderborn.

Gnambs, Timo, und Bernard Batinic. 2010. «Qualitative Online-Forschung». In Handbuch Qualitative Forschung in der Psychologie, herausgegeben von Günter Mey und Katja Mruck, 320-32. Wiesbaden: VS Verlag für Sozialwissenschaften.

Irion, Thomas. 2008. Hypermedia-Recherche im Grundschulalter. Eine qualitative Videostudie zu Vorerfahrungen und Recherchekompetenzen. Baltmannsweiler: Schneider Verlag Hohengehren.

Kelle, Helga. 2001. «Ethnographische Methodologie und Probleme der Triangulation. Am Beispiel der PeerCulture Forschung bei Kindern». Zeitschrift für Soziologie der Erziehung und Sozialisation 21: 192-208.

Kempter, Guido, und Gay Bente. 2004. «Psychophysiologische Wirkungsforschung: Grundlagen und Anwendungen». In Lehrbuch der Medienpsychologie, herausgegeben von Roland Mangold, Peter Vorderer, und Gary Bente, 271-95. Göttingen u.a.: Hogrefe.

Leber, Jasmin, und Irene T. Skuballa. 2014. «Lernräume adaptiv gestalten. Ein blickbewegungsbasierter Ansatz». In Lernräume gestalten - Bildungskontexte vielfältig denken, herausgegeben von Klaus Rummler, 579-91. Münster: Waxmann. 
Marotzki, Winfried. 2003. "Online-Ethnographie - Wege und Ergebnisse zur Forschung im Kulturraum Internet». MedienPädagogik: Zeitschrift für Theorie und Praxis der Medienbildung 3 (Jahrbuch Medienpädagogik): 149-65. https://doi. org/10.21240/mpaed/retro/2017.07.09.X.

Papastefanou, Georgios. 2009. «Ambulatorisches Assessment: Eine Methode (auch) für die Empirische Sozialforschung». In Umfrageforschung, herausgegeben von Martin Weichbold, Johann Bacher, und Christof Wolf. Wiesbaden: VS Verlag für Sozialwissenschaften.

Pelz, Jeff B. 2019. «Eyetracking Research». In Advanced Research Methods for the Socials and Behaviorals Sciences., herausgegeben von John E. Edlund und Austin Lee Nochols, 168-90. Cambridge: University Press.

Richter, Tobias, Johannes Naumann, Marion Brunner, und Ursula Christmann. 2005. «Strategische Verarbeitung Beim Lernen Mit Text Und Hypertext: Strategic Processing in Learning with Text and Hypertext». Zeitschrift Für Pädagogische Psychologie 19 (1/2): 5-22. https://doi.org/10.1024/1010-0652.19.12.5.

Seib, Sibylle. 2006. Internet-Recherche von Grundschulkindern: Eine qualitativ-empirische Studie mit dem Schwerpunkt auf Kindergesprächen. München: Kopaed.

Welker, Martin. 2019. "Computer und onlinegestützte Methoden für die Untersuchung digitaler Kommunikation». In Handbuch Online-Kommunikation, herausgegeben von Wolfgang Schweiger und Klaus Beck, 531-72. Wiesbaden: Springer. 